\section{Lugar de muerte en Chile: un estudio descriptivo de serie temporal entre 1990-2014}

\author{
JORGE BROWNE ${ }^{1, a}$, IGNACIO CASTRO ${ }^{2, b}$, ANDRÉS PAVLOVIC $^{1, \mathrm{a}}$
}

\section{Place of Death in Chile: A descriptive study of a time series from 1990 to 2014}

Background: With the aim to inform end of life public policies, the place of death in Chile, its trends and associated factors were analysed. Materials and Methods: A cross sectional using publically available death database from 1990 to 2014 was conducted. The proportion of hospital deaths was selected as the main outcome. A logistic regression was used to assess the association between place of death, age, and main diagnosis at death. Also, a Prais-Winsten regression and a Chi test were used to assess a time series and regional analysis, respectively. Results: 2,063,615 deaths were analysed. Overall, deaths 898,871 (43.6\%) occurred at hospital. Those who died over 85 years (OR 2,52 IC95\% 2.49-2.55) and those who died from cancer (OR 2.43 IC95\% 2.42-2.45) had higher risk for dying outside de hospital. For the general population and those who die form cancer, there is no evidence for an increase or decrease trend in the proportion of hospital deaths over time ( $p=0,75$ and $p=0.68$, respectively). However, there is an increase of the proportion of hospital deaths in those who died over 85 years ( $p<0.001,27 \%$ in 1990 to 32\% in 2014). Also, there are geographic differences between country regions ( $p<0.001)$ (Eg. Magallanes 52.9\%). Conclusions: The proportion of hospital deaths has been stable over time in the general population and has increased in those over 85 years.

(Rev Med Chile 2019; 147: 727-732)

Key words: Chile; Death; Death Certificates; Terminal Care.
'Sección de Geriatría, División de Medicina, Facultad de Medicina. Pontificia Universidad Católica de Chile. Santiago, Chile. 2Escuela de Salud Pública, Universidad de Chile. Santiago, Chile.

Residente del Programa de Geriatría.

${ }^{\text {b}}$ Residente de Programa de Formación de Especialistas en Salud Pública.

Trabajo no recibió financiamiento.

Los autores declaran no tener conflictos de interés.

Recibido el 30 de enero de 2018, aceptado el 8 julio de 2019.

Correspondencia a:

Dr. Jorge Browne

José Manuel Cousiño 1835,

Providencia. Santiago, Chile. jabrowne@uc.cl hile se encuentra en una transición demográfica avanzada caracterizada por un rápido aumento del número de personas mayores, por lo que se espera que la tasa de mortalidad cruda aumente de manera sostenida hasta el $2050^{1,2}$. Este cambio demográfico podría representar mayores costos en salud ${ }^{3}$, sin embargo, éstos varían según el lugar de cuidado al final de la vida ${ }^{4}$. Además, la congruencia entre el lugar de muerte y la preferencia de las personas se ha sido propuesto como un objetivo deseable en salud ${ }^{5}$.

El lugar de muerte ha sido extensamente estudiado en países de altos ingresos, con resultados heterogéneos. En Europa los porcentajes de muerte hospitalaria van desde 33,9\% (Holanda) a 62,8\% $(\text { Gales })^{6}$. En aquellos países con mayor proporción de muertes hospitalarias, se han realizado políticas públicas específicas para disminuir este porcentaje con resultados favorables (Ej. Inglaterra $)^{7.8}$. Sin embargo, en Canadá, sin esfuerzos específicos también se ha visto una reducción ${ }^{9}$.

En Chile, los estudios que han analizado el lugar de muerte son escasos. En 2007 con el objetivo de describir las causas de muerte en una región en particular, Leiva y León ${ }^{10}$ describieron el lugar de muerte para la población general chilena usando datos entre 1990 y 2003. Sin embargo, hasta donde sabemos no existen estudios que analicen datos actualizados. Además desde el año 2005 las personas con cáncer tienen acceso al plan de Garantías 
Explícitas en Salud (GES) de Cuidados Paliativos ${ }^{11}$. Este plan incluye la posibilidad de cuidados domiciliarios al final de la vida, no obstante el impacto sobre lugar de muerte es incierto.

Con el propósito de informar el diseño de políticas para el final de vida,, este estudio describe el lugar de muerte en Chile y sus cambios en el tiempo.

\section{Métodos}

\section{Diseño y datos}

Se realizó un estudio de corte transversal con datos de mortalidad en Chile obtenidos de la base de datos de defunciones entre los años 1990 y 2014. Estos datos están públicamente disponibles en el Departamento de Estadística e Información en Salud y no permiten identificación personal. En Chile, la información de certificados de defunción tiene $0,06 \%$ de casos faltantes para lugar de muerte y la Organización Panamericana de la Salud ha respaldado una baja proporción de datos con diagnósticos clasificados erróneamente ${ }^{12}$.

Se incluyeron todas las muertes registradas en el período $(\mathrm{n}=2.127 .952)$. Se excluyeron fallecidos menores de un año, dado que representan principalmente muertes neonatales que son menos susceptibles a ser influenciadas por políticas de final de vida. Además se excluyeron los con lugar de defunción ignorado. El lugar de muerte se encuentra descrito como variable categórica incluyendo "Hospital", "Casa" u "Otro", las que fueron reclasificadas en "Hospital” u "Otro" para el análisis. La identificación de pacientes fallecidos por cáncer usó los criterios CIE-9 y 10 correspondientes (CIE-9 140-239; CIE-10: C00-D49). Edad fue transformada en una variable categórica de 5 grupos basados en la distribución poblacional. Considerando una baja frecuencia de muertes no registradas, se consideró que los casos incluidos corresponden a la población total.

El porcentaje de población que muere en hospitales fue considerado el resultado principal y se describieron sus diferencias según edad, región de residencia y diagnóstico. Un test de $\chi^{2}$ fue utilizado para comparar el lugar de muerte en las distintas regiones.

El análisis de serie temporal se analizó mediante un modelo de regresión Prais-Winsten. Se evaluó la presencia de autocorrelación usando el test de Durbin-Watson (DW) y, en caso de estar presente, se utilizo la corrección de Cochrane-Orcutt (CO). Se analizaron tres grupos: población general, aquellos fallecidos por cáncer y mayores a 85 años. Estos dos últimos grupos fueron analizados de manera independiente dado su acceso a servicios de cuidado de final de vida distintos a la población general; los primeros pueden recibir cuidados en domicilio (programa GES), mientras los segundos tienen mayor probabilidad de recibir cuidados de largo plazo de manera institucionalizada ${ }^{13}$.

Se empleo una regresión logística ajustada por género para analizar la asociación de edad y diagnóstico de cáncer con la probabilidad de morir en el hospital. Al comparar grupos de edad, el grupo que falleció entre los 44-55 años fue usado como grupo base, dado que los menores de 40 años solo representan 8,9\% del total de casos. Los datos fueron analizados usando STATA 13.3.

\section{Resultados}

Un total de 2.063.615 casos fueron analizados durante los 25 años observados. Esto corresponde a un promedio de 82.595 muertes anuales (rango: 69.881-100.127 casos anuales); $64.337(3,1 \%)$ casos fueron excluidos por edad ( $<1$ año) y 1.271 $(0,06 \%)$ por datos faltantes. Las regiones Arica y Parinacota y Los Ríos aportan datos a partir del 2007, año en que éstas fueron administrativamente creadas; 947.271 (46\%) casos corresponden a mujeres y la edad media fue de 73 años (p25-p75 60-83 años). Del total, 502.191 (24\%) fallecieron de cáncer.

Durante el período de observación, 898.871 $(43,6 \%)$ personas fallecieron en el hospital. La muerte en hospital es menos frecuente en adultos mayores y en aquellos que fallecen de cáncer (Tabla 1). Al ajustar por género, existe un grado de evidencia alto $(\mathrm{p}<0,001)$ que aquellos que fallecieron después de los 85 años tienen una probabilidad significativamente mayor de morir fuera del hospital que aquellos fallecidos entre los 40 a 55 años (OR 2,52; IC95\%: 2,49-2,55). Además, luego de ajustar por género y edad, existe un grado de evidencia alto $(\mathrm{p}<0,001)$ de que aquellos fallecidos por cáncer tienen una probabilidad significativamente mayor de morir fuera del hospital que aquellos que fallecieron por otras enfermedades (OR 2,43; IC95\%: 2,42-2,45). 
Tabla 1. Lugar de muerte, edad y diagnóstico

\begin{tabular}{|lcc|}
\hline & Hospital & Otro \\
\hline Edad-mediana (RIQ) & $71(58-80)$ & $75(61-84)$ \\
\hline Según grupo edad (\%) & & \\
$\quad<40$ años & 44,4 & 55,6 \\
40-54 años & 48,8 & 51,2 \\
55-69 años & 49,7 & 50,3 \\
70-85 años & 43,9 & 56,1 \\
$>$ >5 años & 30,9 & 69,1 \\
Según diagnóstico (\%) & & \\
Cáncer & 30,1 & 69,9 \\
No cáncer & 47,9 & 52,1 \\
\hline
\end{tabular}

No existe evidencia, tanto en población general como en aquellos fallecidos por cáncer $(\mathrm{p}=0,98$ y $\mathrm{p}=0,63$, respectivamente) que la probabilidad de muerte en el hospital haya cambiado durante el período de observación. Sin embargo, existe alto grado de evidencia $(\mathrm{p}<0,001)$ que aquellos mayores a 85 han aumentado su probabilidad de morir en el hospital durante el período observado. En promedio, este aumento corresponde a un 0,26\% (IC95\%: 0,08 a 0,45\%) anual (Figura 1). En presencia de autocorrelacion (DW 0,79, dL 1.017) esta estimación fue corregida utilizando el método Cochrane-Orcutt.
$\mathrm{Al}$ analizar todo el período de observación se evidenciaron diferencias significativas $(\mathrm{p}<0,001)$ entre la probabilidad de morir según la región de residencia. Aquellos que fallecieron en la región de Los Lagos $(37,8 \%)$ presentan la menor probabilidad de morir en el hospital. Por otro lado, aquellos que fallecieron en la región de Magallanes presentan la mayor probabilidad de morir en el hospital (52,9\%). La Figura 2 describe la probabilidad de morir en el hospital según región de residencia.

\section{Discusión}

Este estudio muestra que el $43 \%$ de las muertes ocurrieron en hospitales, pero aquellos que fallecieron después de los 85 años o por cáncer mantuvieron una menor probabilidad de morir en el hospital durante todo el período observado. A pesar del aumento en el número total de muertes (73.000 en 1990 a 101.100 en 2014) no existe una cambio en el tiempo a incrementar o disminuir el porcentaje de muertes hospitalarias en la población general ni en aquellos que fallecieron por cáncer. Sin embargo, se evidencia un aumento sostenido de la proporción de mayores de 85 años que fallecen en el hospital (27\% el año 1990 y $32 \%$ el año 2014).

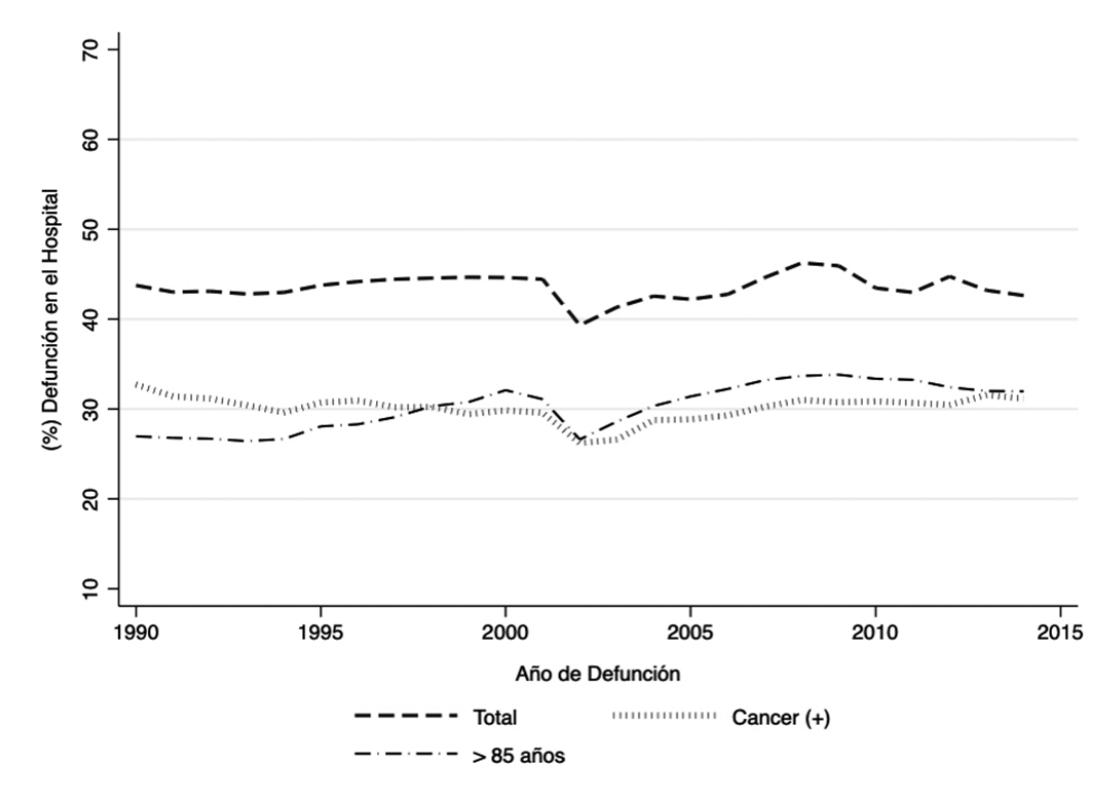

Figura 1. Proporción de muertes hospitalarias en el tiempo, según población total, mayores a 85 años, y aquellos que fallecieron por cáncer. 


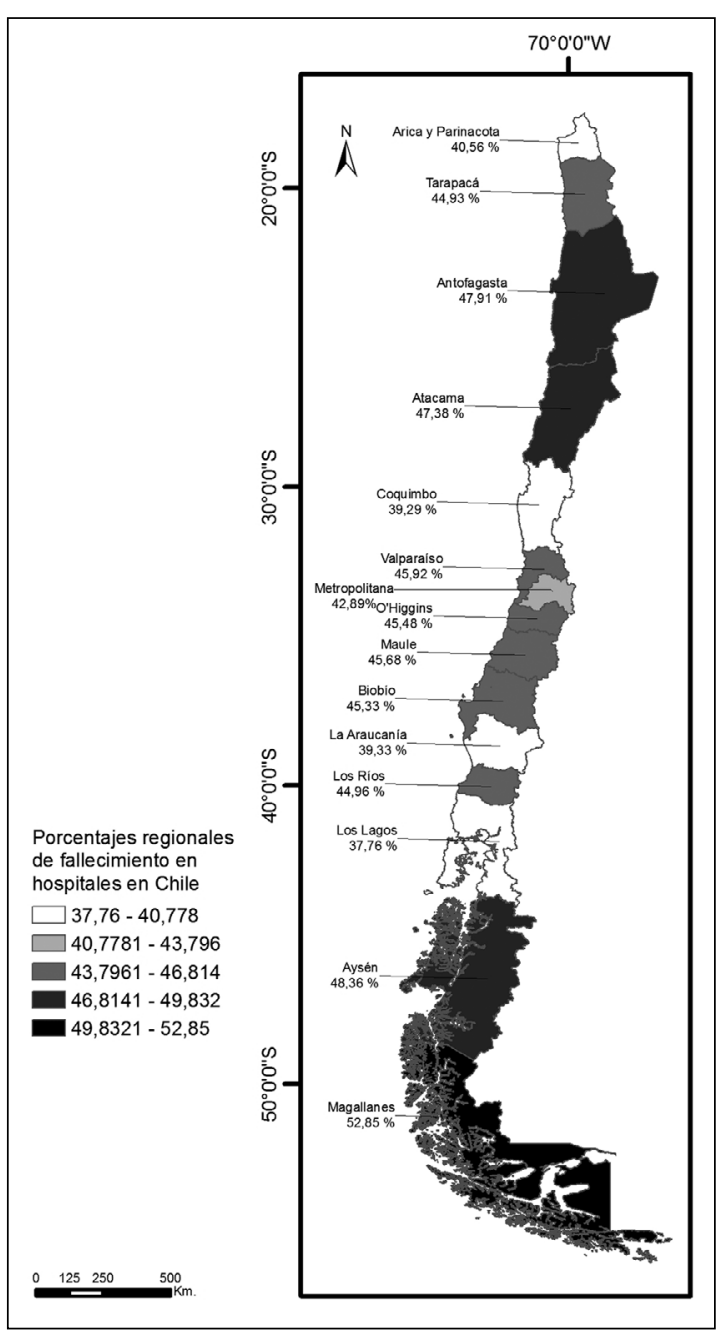

Figura 2. Porcentajes regionales de fallecimiento en hospitales en Chile.

En Chile la proporción de muertes hospitalarias (43\%) se encuentra dentro del rango descrito en la literatura, pero es significativamente menor a lo actualmente descrito en una gran mayoría de países de altos ingresos como Suecia (62\%), Inglaterra (58\%), Estados Unidos de Norteamérica $(52 \%)$ y Bélgica $(51 \%)^{6,14}$. Si bien esta comparación no contempla ajustes por edad, la proporción de adultos mayores que muere en hospitales es menor al resto de la población. Por tanto, una mayor proporción de muertes hospitalarias no se explicaría por envejecimiento poblacional. En Noruega se ha descrito una disminución significativa de las muertes hospitalarias en las últimas décadas (42\% en 1987 a $34 \%$ en 2011 ) que ha sido reemplazado por un mayor número de muertes en residencias de larga estadía (29,5\% a 45,5\% durante el mismo período $)^{15}$. El caso de Noruega no es aislado y asemeja a lo ocurrido en otros países como Bélgica, Reino Unido y Canadá, entre otros $^{16}$. En estos casos, la disminución en la proporción de muertes hospitalarias se ha atribuido a políticas públicas dirigidas a favorecer muertes en casa y lugares de larga estadía ${ }^{16}$. En Chile la proporción de muertes en el hospital es comparativamente baja, pero con una tendencia en el tiempo estacionaria para la población general, e incluso con aumento en mayores de 85 años, lo que se contrapone con las tendencias de países de mayores ingresos descritas previamente. Un aumento de muertes hospitalarias se ha descrito en otros países con poblaciones envejecidas, como Portugal, donde no se han implementado políticas de final de vida con impacto en lugar de muerte ${ }^{17}$.

Morir fuera del hospital se ha asociado a mejoras en calidad de vida al final, menores costos para el sistema de salud y mayor concordancia con preferencias de lugar de muerte. En un análisis retrospectivo a familiares de fallecidos en Inglaterra, Fleming y colaboradores ${ }^{18}$ describen que aquellos que fallecieron en lugares de larga estadía tienen cuatro veces mayor probabilidad de morir de manera confortable comparados con aquellos que fallecieron en el hospital. Por otro lado, se ha descrito una mayor preferencia para morir en residencias y en casa, especialmente en personas mayores, donde hasta el $86 \%$ lo prefiere $^{19}$. Finalmente, aquellos que mueren en lugares de larga estadía incurren en gastos en salud significativamente menores comparados a aquellos que mueren en el hospital. Residir en instituciones de larga estadía disminuye el riesgo de hospitalizaciones en los últimos 30 días previos al fallecimiento ${ }^{20}$. Incluso se ha llegado a estimar que aquellos que fallecen en residencias de larga estadía incurren en la mitad de los gastos de salud que aquellos que fallecen en el hospital ${ }^{20}$.

En Chile la tendencia de muerte en lugares de larga estadía durante el período observado es incierta. Los certificados de muerte tabulan lugar de muerte en "casa", "hospital" y "otro" donde no se especifica si "casa" corresponde a domicilio personal o lugar de larga estadía. Sin embargo, como la proporción de muertes hospitalarias se ha mantenido durante el período de observación es 
improbable un incremento significativo de muertes en estos lugares. Más aun, en aquellos mayores de 85 años que reportan mayores preferencias para morir fuera del hospital y tienen mayor probabilidad de estar institucionalizados, la probabilidad de morir en el hospital ha aumentado de manera sostenida. Por otro lado, durante el período de observación no disminuyó la proporción de muertes hospitalarias en aquellos con cáncer, pese que el 2005 se garantizara el acceso y oportunidad a cuidados paliativos domiciliarios a este subgrupo poblacional. Esto sugiere que en Chile el impacto de los lugares de larga estadía y cuidados domiciliarios sobre el lugar de muerte es débil cuando se compara con las tendencias descritas en otros países de mayores ingresos.

La interpretación de las diferencias en lugar de muerte según área geográfica, edad y diagnóstico es compleja. El lugar de muerte depende de múltiples factores tales como, acceso a cuidados de final de vida, preferencias personales y cuidadores y complejidad de cuidados. Las diferencias encontradas a nivel poblacional hacen suponer que existen factores estructurales distintos al azar que determinan el lugar de muerte en algunas subpoblaciones. Estas diferencias pudieran ser apropiadas, o bien, representar necesidades no cubiertas de cuidados al final de vida. Chile ha implementado programas de cuidados de largo plazo, desarrollando Establecimientos de Larga Estadía para el Adulto Mayor (ELEAM) y asegurado la garantía de acceso, oportunidad y protección financiera para servicios de cuidados paliativos. Sin embargo, se ha sugerido que el financiamiento para los ELEAM es insuficiente y la cobertura de cuidados paliativos garantizados por GES excluye a pacientes que presentan patologías no oncológicas ${ }^{21,22}$.

Las proyeciones del Instituto Nacional de Estadísticas estiman que la tasa cruda de mortalidad continuará aumentando llegando a 219.800 muertes/año al 2050, lo que representa un aumento casi el doble respecto al actual (104.026 muertes durante 2016). En ausencia de sistemas de atención domiciliaria y programas de largo plazo robustos nacionales que permitan modificar la tendencia de distribución del lugar de muerte, el aumento en la tasa cruda de mortalidad implicará un aumento en la carga hospitalaria. Por otro lado, los resultados de este estudio obligan a entender la calidad del proceso de muerte fuera de los hospitales en Chile.
En aquellos que mueren en el hospital teóricamente sus necesidades en salud, como manejo del dolor, debieran estar cubiertas. Pero para el 57\% de los fallecidos fuera de éste, la cobertura de estas necesidades son inciertas. Esto es especialmente importante en subpoblaciones que tienen mayor riesgo de morir fuera del hospital, tales como los adultos mayores.

\section{Fortalezas y debilidades}

Al usar certificados de defunción oficiales y de acceso público, este estudio fue capaz de obtener los datos de toda la población durante un período de tiempo extenso. Además, la baja proporción de casos con lugar de muerte desconocida disminuye el riesgo de sesgo de selección. Sin embargo, existen limitaciones que deben ser tomadas en cuenta.

Existen factores no considerados tales como voluntades anticipadas, acceso a centros de salud $\mathrm{y}$ red de apoyo familiar que pudieran determinar el lugar de muerte. Una limitada disponibilidad de camas, por ejemplo, pudiera aumentar la probabilidad de que éstas sean priorizadas a personas de mayor expectativa de vida. Por lo que las diferencias descritas por edad, diagnóstico y lugar geográfico son susceptibles a variables confundentes que impiden la atribución de causalidad.

La asociación entre lugar de muerte e implementación de Cuidados Paliativos en el plan GES debe ser interpretada cuidadosamente. Este estudio postula que la implementación de esta política aumentaría la cobertura $y$, por tanto, podría esperarse una caída en la probabilidad de muerte en el hospital en fallecidos por cáncer. Sin embargo, se desconoce si los objetivos de los programas locales de cuidados paliativos están orientados a favorecer la muerte en casa. Estos resultados solo permiten concluir que posterior a la implementación de esta política, el lugar de muerte no ha variado en aquellos fallecidos por cáncer. Una evaluación en profundidad de esta política está fuera de los objetivos de este estudio.

\section{Conclusiones e implicancias para política pública}

A diferencia de países de mayores ingresos donde se han implementado políticas de final de vida que han disminuido la proporción de muertes hospitalarias, en Chile esta proporción se ha 
mantenido e incluso aumentado en los mayores a 85 años. Estos resultados sugieren que en las próximas décadas es esperable que aumenten en costos en salud al final de vida y, posiblemente, disminuya la concordancia con las preferencias de la población sobre lugar de muerte. Cambios en recolección de datos, como la inclusión de cuidados de largo plazo como lugar de muerte en certificados de defunción, y evaluación de preferencias al final de la vida son necesarias para el diseño de políticas dirigidas al buen morir que respondan a las necesidades en salud, estén alineadas con las preferencias de la población, y sean económicamente sustentables.

Agradecimientos: A la Dra. Marcela Carrasco por el constante apoyo y guía durante este trabajo.

\section{Referencias}

1. OCDE Health Data. Demografic Refecences 2017 [Available from: http://stats.oecd.org/Index.aspx?DataSetCode=HEALTH_DEMR [Accedido el 28 de julio de 2017].

2. Instituto Nacional de Estadística. Chile: Proyecciones y Estimaciones de Población. Total País Período de Información: 1950-2050. Santiago, Chile: INE, 2005.

3. Davis MA, Nallamothu BK, Banerjee M, Bynum JPW. Identification of four unique spending patterns among older adults in the last year of life challenges standard assumptions. Health Af (Project Hope) 2016; 35 (7): 1316-23.

4. Pham B, Krahn M. End-of-Life Care Interventions: An Economic Analysis. Ont Health Technol Assess Ser 2014; 14 (18): 1-70.

5. Gomes B, Calanzani N, Gysels M, Hall S, Higginson IJ. Heterogeneity and changes in preferences for dying at home: A systematic review. BMC Palliative Care 2013; 12: 7 .

6. Cohen J, Bilsen J, Addington-Hall J, Lofmark R, Miccinesi G, Kaasa S, et al. Population-based study of dying in hospital in six European countries. Palliat Med 2008; 22 (6): 702-10.

7. Gao W, Ho YK, Verne J, Glickman M, Higginson IJ. Changing patterns in place of cancer death in England: a population-based study. PLoS Med 2013; 10 (3): e1001410.

8. Barratt H, Asaria M, Sheringham J, Stone P, Raine R, Cookson R. Dying in hospital: socioeconomic inequality trends in England. J Health Serv Res Policy 2017;

\section{7.}

9. Wilson DM, Truman CD, Thomas R, Fainsinger R, Kovacs-Burns K, Froggatt K, et al. The rapidly changing location of death in Canada, 1994-2004. Soc Sci Med 2009; 68 (10): 1752-8.

10. Leiva HH, León KF. [Medical coverage of the disease that causes death and place of death in the sixth region of Chile from 1990 to 2003]. Rev Med Chile 2007; 135 (8): 1025-33.

11. Valdivieso C, Montero J. El plan AUGE: 2005 al 2009. Rev Med Chile 2010; 138: 1040-6.

12. Pan American Health Organization. Health in the Americas 2007. Washington, DC: Pan American Health Organization, 2007.

13. Fleming J, Calloway R, Perrels A, Farquhar M, Barclay S, Brayne C, et al. Dying comfortably in very old age with or without dementia in different care settings - a representative "older old" population study. BMC Geriatr 2017; 17 (1): 222.

14. Gruneir A, Mor V, Weitzen S, Truchil R, Teno J, Roy J. Where people die: a multilevel approach to understanding influences on site of death in America. Medical care research and review: MCRR 2007; 64 (4): 351-78.

15. Kalseth J, Theisen OM. Trends in place of death: The role of demographic and epidemiological shifts in endof-life care policy. Palliat Med 2017; 31 (10): 964-74.

16. Gomes B, Calanzani N, Higginson IJ. Reversal of the British trends in place of death: time series analysis 2004-2010. Palliat Med 2012; 26 (2): 102-7.

17. Sarmento VP, Higginson IJ, Ferreira PL, Gomes B. Past trends and projections of hospital deaths to inform the integration of palliative care in one of the most ageing countries in the world. Palliat Med 2016; 30 (4): 363-73.

18. Fleming J, Calloway R, Perrels A, Farquhar M, Barclay $S$, Brayne C. Dying comfortably in very old age with or without dementia in different care settings - a representative "older old" population study. BMC Geriatr 2017; 17 (1): 222.

19. Davidson S, Gentry T. End of Life Evidence Review. London: Age UK. 2013.

20. Miller SC, Gozalo P, Mor V. Hospice enrollment and hospitalization of dying nursing home patients. Am J Med 2001; 111 (1): 38-44.

21. Matus-López M, Cid Pedraza C. Building Long-Term Care Policies in Latin America: New Programs in Chile. J Am Med Dir Assoc 2015; 16 (10): 900 e7-10.

22. Instituto Nacional de Estadística. Defunciones y Mortalidad por causas 2017 [Available from: http://www.deis. $\mathrm{cl}$ /defunciones-y-mortalidad-por-causas/ [Accedido el 4 de septiembre de 2017]. 